\title{
Multi-reference perturbation theory study on the CsYb molecule including the spin-orbit coupling
}

\author{
Darya N. Meniailava ${ }^{a}$, Maksim B. Shundalau ${ }^{\mathrm{a}, \mathrm{b}, *}$ \\ ${ }^{a}$ Physics Department, Belarusian State University, 4 Nezaležnaści Ave., 220030 Minsk, Belarus \\ ${ }^{\mathrm{b}}$ A.N. Sevchenko Institute of Applied Physical Problems at Belarusian State University, 7 Kurčataǔ Str., 220108 Minsk, Belarus
}

\section{A R T I C L E I N F O}

\section{Article history:}

Received 22 February 2017

Received in revised form 27 March 2017

Accepted 28 March 2017

Available online 5 April 2017

\section{Keywords:}

Multi-reference perturbation theory

calculations

CsYb molecule

Potential energy curves

Spin-orbit coupling

Vibrational states

Franck-Condon factors

\begin{abstract}
A B S T R A C T
We present CASSCF/XMCQDPT2 level of theory calculations of the ground and sixteen low-lying excited electronic states of the $\mathrm{CsYb}$ molecule taking into account the spin-orbit coupling. Spectroscopic constants (electronic term energies, equilibrium internuclear distances, dissociation energies, harmonic vibrational frequencies), transition dipole moments, Franck-Condon factors and vibrational energies of the CsYb molecule have been obtained. The energies of the ground and first exited states at the asymptotic limits definitely satisfy the experimental data for cesium and ytterbium atoms. All the data obtained allow to predict and realize two-photon schemes for producing ultracold CsYb molecules and carry out spectral experiments with them.
\end{abstract}

(c) 2017 Elsevier B.V. All rights reserved.

\section{Introduction}

Nowadays in the field of cold and ultracold molecules theoretical and practical studying of a new class of heteronuclear diatomic molecules, which include an atom of alkali metals and one of transition metals, is of great interest. In a diatomic molecule consisting of an alkali metal atom and, for example, of an atom of the lanthanide series with even atomic number one of the electrons is found out to be unpaired. This means that such a molecule besides a permanent electric dipole moment also has a permanent magnetic dipole moment. Thus, it is possible to effectively manipulate of the molecular quantum matter by external magnetic field. Moreover these molecules offer the possibilities for high precision measurements of fundamental constants, for quantum many-body physics and quantum information, for testing fundamental models, etc. $[1,2]$. The CsYb molecule is proposed to be an attractive candidate for the possibilities mentioned. For efficient production of ultracold $\mathrm{CsYb}$ molecules by two-photon schemes the knowledge of exact potential energy curves (PECs), vibrational energies, molecular spectroscopic and dynamic parameters are crucial.

Up to our knowledge, no experimental data are evaluable for a system of electronic states of the CsYb molecule, and there are only

\footnotetext{
* Corresponding author at: Physics Department, Belarusian State University, 4 Nezaležnaści Ave., 220030 Minsk, Belarus.

E-mail address: shundalov@bsu.by (M.B. Shundalau).
}

three $a b$ initio calculations for the ground $X^{2} \Sigma^{+}$state in $\operatorname{CCSD}(\mathrm{T})$ (Coupled Cluster) [3] and CASSCF/MRCI (Complete Active Space Self-Consistent Field/Multi-Reference Configuration Interaction) approximation $[4,5]$ respectively, and for the first excited states (namely spin-free $1^{2} \Pi$ and $2^{2} \Sigma^{+}$states), performed by Meyer and Bohn [4] at the CASSCF/MRCI level of theory. The results of these calculations for the ground state are quite different: $R_{e}=5.657 \AA$, $D_{e}=182 \mathrm{~cm}^{-1} \quad[4] ; \quad R_{e}=5.161 \AA, \quad D_{e}=542 \mathrm{~cm}^{-1} \quad$ [5] and $R_{e}=5.144 \AA, D_{e}=621 \mathrm{~cm}^{-1}$ [3] (where $R_{e}$ is equilibrium internuclear distance, $D_{e}$ is dissociation energy). In this connection it is important to compare the calculated characteristics of other alkali-metal-ytterbium diatomic molecules: LiYb, NaYb, KYb, and RbYb.

The dissociation energies calculated at the different levels of theory for the alkali-metal-ytterbium diatomics are: $1151 \mathrm{~cm}^{-1}$ (Tohme et al. [6], MRCI), $1403 \mathrm{~cm}^{-1}$ (Shao et al. [5], MRCI), 1422 and $1490 \mathrm{~cm}^{-1}$ (Gopakumar et al. [7], CASPT2), $1577 \mathrm{~cm}^{-1}$ (Zhang et al. [8], $\operatorname{CCSD}(\mathrm{T})$ ), $1641 \mathrm{~cm}^{-1}$ (Brue and Hutson [3], $\operatorname{CCSD}(\mathrm{T})$ ), $2289 \mathrm{~cm}^{-1}$ (Kotochigova et al. [9], $\operatorname{CCSD}(\mathrm{T})$ ) for the LiYb molecule; 248-1291 $\mathrm{cm}^{-1}$ (Tohme and Korek [10], MRCI), $912 \mathrm{~cm}^{-1}$ (Shao et al. [5], MRCI), $1012 \mathrm{~cm}^{-1}$ (Brue and Hutson [3], $\operatorname{CCSD}(\mathrm{T})$ ) for the NaYb molecule; $696 \mathrm{~cm}^{-1}$ (Shao et al. [5], MRCI), $737 \mathrm{~cm}^{-1}$ (Brue and Hutson [3], CCSD (T)), 858 and $971 \mathrm{~cm}^{-1}$ (Tohme and Korek [11], MRCI) for the KYb molecule; and $193 \mathrm{~cm}^{-1}$ (Meyer and Bohn [4], MRCI), $531 \mathrm{~cm}^{-1}$ (Shundalau and Minko [12], XMCQDPT2), $565 \mathrm{~cm}^{-1}$ (Shao et al. [5], MRCI), 591-868 $\mathrm{cm}^{-1}$ 
(Sørensen et al. [13] MRCI and CCSD), $656 \mathrm{~cm}^{-1}$ (Brue and Hutson [3], $\operatorname{CCSD}(\mathrm{T})), \sim 700 \mathrm{~cm}^{-1}$ (Tohme and Korek [14], MRCI), 704 and $786 \mathrm{~cm}^{-1}$ (Borkowski et al. [15], CCSD(T)) for the RbYb molecule. So, one can see that different multi-reference methods yield different results for all alkali-metal-ytterbium diatomics, and there is a trend of decreasing of the dissociation energies in the LiYb, NaYb, $\mathrm{KYb}$, and $\mathrm{RbYb}$ molecules row.

Turning back to the CsYb molecule it is worth mentioning that the calculations by Shao et al. [5] $\left(D_{e}=542 \mathrm{~cm}^{-1}\right)$ and Brue and Hutson [3] $\left(D_{e}=621 \mathrm{~cm}^{-1}\right)$ were performed only for the ground state, i.e. without state averaged (SA) procedure on ground and some excited states. It means that the influence of the excited states on form and depth of the ground state well through density matrix averaging was absent. Note that for the weakly-bounded systems where the depth well is small (CsYb case), such influence might play an important role in forming of the ground state potential energy function.

Lately MRPT calculations at the CASSCF/XMCQDPT2 (Extended Multi-Configuration Quasi-Degenerate 2nd Order Perturbation Theory) [16] level of theory were performed for the KRb [17] and RbYb [12] molecules. In study [17] we obtained the spectroscopic parameters of the ground state for the KRb molecule with a high accuracy. As a result of calculations in [12] the energies obtained for the first two asymptotic limits exactly respond to the experimental data for rubidium and ytterbium atoms. An explanation of such sufficient results of the calculations is also discussed in [12]. Thus we use the same technique expecting to achieve the best results for $\mathrm{CsYb}$ molecule.

The aim of this work is to carry out ab initio calculations of PECs of the low-lying electronic states taking into account the spin-orbit coupling (SOC), to determine molecular spectroscopic constants, vibrational energies, Franck-Condon factors (FCFs) for the vibronic transitions of diatomic polar $\mathrm{CsYb}$ molecule at the high level of theory. Particular attention is paid to the ground $X^{2} \Sigma_{1 / 2}^{+}$state and lowlying excited $1^{2} \Pi_{1 / 2}, 1^{2} \Pi_{3 / 2}$, and $2^{2} \Sigma_{1 / 2}^{+}$states.

\section{Computations}

The electronic configuration and the ground state term for the cesium atom are $[\mathrm{Xe}] 6 \mathrm{~s}$ and ${ }^{2} \mathrm{~S}_{1 / 2}$, respectively. The first three excited configurations, atomic terms and corresponding to them energies are: $\quad 5 \mathrm{p}^{6} 6 \mathrm{p} \rightarrow{ }^{2} \mathrm{P}_{1 / 2} \quad\left(11178.268 \mathrm{~cm}^{-1}\right), \quad{ }^{2} \mathrm{P}_{3 / 2}$ $\left(11732.308 \mathrm{~cm}^{-1}\right) ; \quad 5 p^{6} 5 \mathrm{~d} \rightarrow{ }^{2} \mathrm{D}_{3 / 2} \quad\left(14499.358 \mathrm{~cm}^{-1}\right), \quad{ }^{2} \mathrm{D}_{5 / 2}$ $\left(14596.842 \mathrm{~cm}^{-1}\right)$, and $5 \mathrm{p}^{6} 7 \mathrm{~s} \rightarrow{ }^{2} \mathrm{~S}_{1 / 2}\left(18535.529 \mathrm{~cm}^{-1}\right)$ [18]. The SOC splittings of the ${ }^{2} \mathrm{P}$ and ${ }^{2} \mathrm{D}$ terms are 554.040 and $98.484 \mathrm{~cm}^{-1}$, respectively.

The electronic configuration and the ground state term for the ytterbium atom are $[\mathrm{Xe}] 4 \mathrm{f}^{14} 6 \mathrm{~s}^{2}$ and ${ }^{1} \mathrm{~S}_{0}$, respectively. The first excited configuration, appropriate triplet and singlet terms, and energies of the states for the $\mathrm{Yb}$ atom are: $4 \mathrm{f}^{14} 6 \mathrm{~s} 6 \mathrm{p} \rightarrow{ }^{3} \mathrm{P}_{0}$ $\left(17288.499 \mathrm{~cm}^{-1}\right),{ }^{3} \mathrm{P}_{1}\left(17992.007 \mathrm{~cm}^{-1}\right),{ }^{3} \mathrm{P}_{2}\left(19710.388 \mathrm{~cm}^{-1}\right)$, and ${ }^{1} \mathrm{P}\left(25068.222 \mathrm{~cm}^{-1}\right)$ [18]. The SOC splittings of the triplet ${ }^{3} \mathrm{P}$ term are 703.508 and $1718.381 \mathrm{~cm}^{-1}$.

The next excited term $(7 / 2,3 / 2)_{2,3,4,5}$ originates from the $4 \mathrm{f}^{13} 5 \mathrm{~d}^{1} 6 \mathrm{~s}^{2}$ excited configuration with energies of the states lying in the range of $23288-28184 \mathrm{~cm}^{-1}$ [18]. The triplet ${ }^{3} \mathrm{D}_{1,2,3}$ term arising from the $4 \mathrm{f}^{14} 5 \mathrm{~d} 6 \mathrm{~s}$ configuration with the energies ranging $24489-25271 \mathrm{~cm}^{-1}$ [18] and the ${ }^{1} \mathrm{P}$ term (see above) are located between the components of the $(7 / 2,3 / 2)_{2,3,4,5}$ term. Therefore calculations of the molecular electronic states corresponding to given or higher excited configurations of the $\mathrm{Yb}$ atom require including $\mathrm{f}$ electrons into the active space. The states just mentioned are not considered here.

We carry out the PECs calculations for spin-free (doublet and quartet) and spin-mixed states corresponding to the five lowest dissociation limits: $C s(6 s)+Y b\left(6 s^{2}\right)$ (limit I), $C s(6 p)+Y b\left(6 s^{2}\right)$ (limit II), $\mathrm{Cs}(5 \mathrm{~d})+\mathrm{Yb}\left(6 \mathrm{~s}^{2}\right)$ (limit III), Cs(7s) $+\mathrm{Yb}\left(6 \mathrm{~s}^{2}\right)$ (limit IV), Cs(6s) $+\mathrm{Yb}(6 \mathrm{~s} 6 \mathrm{p})$ (limit V). It is worth mentioning that ${ }^{2} \mathrm{~S}_{1 / 2}$ term of the Cs atom occurs between the ${ }^{3} \mathrm{P}_{1}$ and ${ }^{3} \mathrm{P}_{2}$ components of the ${ }^{3} \mathrm{P}$ term of the $\mathrm{Yb}$ atom. The molecular electronic terms relating to the $\mathrm{Cs}$ $(5 d)+Y b\left(6 s^{2}\right)$ and $C s(7 s)+Y b\left(6 s^{2}\right)$ dissociation limits are supposed to be highly perturbed by the terms relating to the $\mathrm{Cs}(6 \mathrm{~s})+\mathrm{Yb}$ (6s6p) dissociation limit. As we do not take into account during the calculations the terms corresponding to the $\mathrm{Cs}(6 \mathrm{~s})+\mathrm{Yb}$ $\left(4 \mathrm{f}^{13} 5 \mathrm{~d}^{1} 6 \mathrm{~s}^{2}\right)$ limit, situated much more above just mentioned terms, the terms corresponding to the $\mathrm{Cs}(5 \mathrm{~d})+\mathrm{Yb}\left(6 \mathrm{~s}^{2}\right)$ dissociation limit lift up and the term corresponding to the $\mathrm{Cs}(7 \mathrm{~s})+\mathrm{Yb}\left(6 \mathrm{~s}^{2}\right)$ dissociation limit is pushed out. Consequently, if excitations of the felectrons of the $\mathrm{Yb}$ atom are not taken into account in the active space, PECs of these terms $[6,10,11,14]$ will be of the artifact character

The asymptotic limit I comprises the ground $X^{2} \Sigma^{+}$state, the asymptotic limit II comprises the doublet $2^{2} \Sigma^{+}$and $1^{2} \Pi$ states, the asymptotic limit III comprises the doublet $3^{2} \Sigma^{+}, 2^{2} \Pi$, and $1^{2} \Delta$ states, the asymptotic limit IV comprises only one doublet $4^{2} \Sigma^{+}$state, and the asymptotic limit $\mathrm{V}$ comprises the doublet $5^{2} \Sigma^{+}, 3^{2} \Pi$ states, and quartet $1^{4} \Sigma^{+}, 1^{4} \Pi$ states. As one takes into account spin-orbit effects the $X^{2} \Sigma^{+}, 2^{2} \Sigma^{+}, 3^{2} \Sigma^{+}, 4^{2} \Sigma^{+}$, and $5^{2} \Sigma^{+}$ states transform into the $X^{2} \Sigma_{1 / 2}^{+}, 2^{2} \Sigma_{1 / 2}^{+}, 3^{2} \Sigma_{1 / 2}^{+}, 4^{2} \Sigma_{1 / 2}^{+}$, and $5^{2} \Sigma_{1 / 2}^{+}$ states, respectively; the $1^{2} \Pi, 2^{2} \Pi$, and $3^{2} \Pi$ states split into the $1^{2} \Pi_{3 / 2}, 1^{2} \Pi_{1 / 2}, 2^{2} \Pi_{3 / 2}, 2^{2} \Pi_{1 / 2}$ and $3^{2} \Pi_{3 / 2}, 3^{2} \Pi_{1 / 2}$ states, respectively; the $1^{4} \Sigma^{+}$state splits into the $1^{4} \Sigma_{3 / 2}^{+}$and $1^{4} \Sigma_{1 / 2}^{+}$states; the $1^{4} \Pi$ state splits into the $1^{4} \Pi_{5 / 2}, 1^{4} \Pi_{3 / 2}, 1^{4} \Pi_{1 / 2}$, and $1^{4} \Pi_{-1 / 2}$ states.

It is typical for heavy diatomic molecules containing an alkali metal to use $(n) \Omega$ notation, as spin-mixed states arise from interaction between the states with the different spin. But it is worth using ${ }^{(2 S+1)} \Lambda_{\Omega}$ notation to lay stress on the relationship between the spin-mixed and the spin-free states. Both notations $\left({ }^{(2 S+1)} \Lambda_{\Omega}\right.$ and $(n) \Omega$ ) are given in Table 1 .

We used Stuttgart fully relativistic large core effective core potential (ECP) for ytterbium [19] and Stuttgart fully relativistic small core ECP for cesium [20] atoms during the calculations. The corresponding Gaussian basis sets are (7s7p6d) (ECP60MDF) for ytterbium atom [19] and $(12 \mathrm{~s} 11 \mathrm{p} 5 \mathrm{~d} 3 \mathrm{f} 2 \mathrm{~g}) \rightarrow[8 \mathrm{~s} 8 \mathrm{p} 5 \mathrm{~d} 3 \mathrm{f} 2 \mathrm{~g}]$ (ECP46MDF) for cesium atom [20].

The CASSCF calculations were done for active space including 3 electrons in 12 orbitals. The SA-procedure was realized for 11 doublet and 3 quartet states. The $\operatorname{SA}-\operatorname{CASSCF}(3,12)$ calculations were carried out pointwisely by steps of $0.1,0.05,0.25$, and $0.50 \AA$ for the internuclear distances ranging 3.50-4.10, 4.10-6.10, 6.1010.00 , and $10.00-17.00 \AA$, respectively.

To obtain more reliable energies XMCQDPT2 method [16] was used. All the eight lowest double occupied orbitals were involved in the perturbation calculations, the ISA shift [21] of 0.190 [22] being used. The dimensions of the effective Hamiltonian were $11 \times 11$ and $5 \times 5$ for doublet and quartet states, respectively.

For the spin-orbit coupling (SOC) calculations we used the oneelectron Pauli-Breit operator. The effective nuclear charges $Z_{\text {eff, }}$, which always turn out to be of high value due to errors coursed by nodeless ECPs wave functions (for details see [23-25]), for $\mathrm{Yb}$ and Cs were 7137.0 and 7965.0 respectively.

We obtained potential curves for $\mathrm{CsYb}$ using the Firefly quantum chemical package [26]. For determining the electronic energy terms $T_{e}$ and the equilibrium internuclear distances $R_{e}$ we used the fourth degree polynomial approximation of the $a b$ initio PECs near minima.

The calculations of transition dipole moments (TDMs) are performed at the CASSCF/XMCQDPT2 + SOC level of theory using GAMESS suit of codes [27]. 
Table 1

Molecular spectroscopic constants of ${ }^{133} \mathrm{Cs}^{174} \mathrm{Yb}$ molecule.

\begin{tabular}{|c|c|c|c|c|c|c|c|}
\hline \multicolumn{2}{|l|}{ Term } & \multirow[t]{2}{*}{$T_{e}, \mathrm{~cm}^{-1}$} & \multirow[t]{2}{*}{$R_{e}, \AA$} & \multirow[t]{2}{*}{$\omega_{e}, \mathrm{~cm}^{-1}$} & \multirow[t]{2}{*}{$D_{e}, \mathrm{~cm}^{-1}$} & \multicolumn{2}{|c|}{ Asymptotic limit, $\mathrm{cm}^{-1}$} \\
\hline${ }^{(2 S+1)} \Lambda_{\Omega}$ & $(n) \Omega$ & & & & & Experimental $^{\mathrm{a}}$ & Calculated \\
\hline$X^{2} \Sigma_{1 / 2}^{+}$ & $(1) 1 / 2$ & $\begin{array}{l}-158.7 \\
-182^{\mathrm{b}} \\
-621^{\mathrm{c}} \\
-542^{\mathrm{d}}\end{array}$ & $\begin{array}{l}5.763 \\
5.66^{\mathrm{b}} \\
5.144^{\mathrm{c}} \\
5.161^{\mathrm{d}}\end{array}$ & $\begin{array}{l}12.1 \\
9.9^{\mathrm{b}} \\
19.56^{\mathrm{d}}\end{array}$ & $\begin{array}{l}158.7 \\
182^{\mathrm{b}} \\
621^{\mathrm{c}} \\
542^{\mathrm{d}}\end{array}$ & 0.0 & 0.0 \\
\hline $1^{2} \Pi_{1 / 2}$ & $(2) 1 / 2$ & 6262.4 & $\begin{array}{l}4.051 \\
4.18^{\mathrm{b}}\end{array}$ & $\begin{array}{l}60.0 \\
50.9^{\mathrm{b}}\end{array}$ & $\begin{array}{l}4915.4 \\
2710^{b}\end{array}$ & 11178.0 & 11177.8 \\
\hline $1^{2} \Pi_{3 / 2}$ & $(1) 3 / 2$ & 7425.2 & $\begin{array}{l}4.100 \\
4.18^{\mathrm{b}}\end{array}$ & $\begin{array}{l}58.6 \\
50.9^{\mathrm{b}}\end{array}$ & $\begin{array}{l}4307.1 \\
2710^{\mathrm{b}}\end{array}$ & 11728.9 & 11728.7 \\
\hline $2^{2} \Sigma_{1 / 2}^{+}$ & (3) $1 / 2$ & 9908.2 & $\begin{array}{l}4.932 \\
4.98^{\mathrm{b}}\end{array}$ & $\begin{array}{l}41.2 \\
20.9^{\mathrm{b}}\end{array}$ & $\begin{array}{l}1820.5 \\
490^{b}\end{array}$ & & 11732.3 \\
\hline $2^{2} \Pi_{1 / 2}$ & $(4) 1 / 2$ & 12866.6 & 4.858 & 24.9 & 1196.5 & 14499.3 & 14063.1 \\
\hline $2^{2} \Pi_{3 / 2}$ & $(2) 3 / 2$ & 13502.0 & 4.777 & 19.8 & 1256.7 & 14596.8 & 14758.7 \\
\hline $3^{2} \Sigma_{1 / 2}^{+}$ & (5) $1 / 2$ & 13455.2 & 4.220 & 45.1 & 2339.2 & & 15794.4 \\
\hline $4^{2} \Sigma_{1 / 2}^{+}$ & (6) $1 / 2$ & 15074.5 & 5.675 & 14.6 & 721.0 & 18535.5 & 15795.5 \\
\hline $3^{2} \Pi_{1 / 2}$ & (7) $1 / 2$ & 14830.5 & 4.233 & 55.0 & 2204.2 & 17288.5 & 17034.7 \\
\hline $1^{4} \Pi_{-1 / 2}$ & $(1)-1 / 2$ & 15818.0 & 4.453 & 53.6 & 2023.2 & 17992.0 & 17841.2 \\
\hline $1^{4} \Pi_{1 / 2}$ & $(8) 1 / 2$ & 16247.7 & 4.561 & 36.9 & 1594.7 & & 17842.4 \\
\hline $3^{2} \Pi_{3 / 2}$ & (3) $3 / 2$ & 16559.6 & 4.568 & 35.0 & 1283.3 & & 17842.9 \\
\hline $5^{2} \Sigma_{1 / 2}^{+}$ & (9) $1 / 2$ & 16146.4 & 4.875 & 55.4 & 3308.6 & 19710.4 & 19455.0 \\
\hline $1^{4} \Pi_{3 / 2}$ & $(4) 3 / 2$ & 16549.5 & 4.478 & 54.5 & 2905.8 & & 19455.3 \\
\hline $1^{4} \Sigma_{1 / 2}^{+}$ & $(10) 1 / 2$ & 18673.0 & 5.675 & 33.6 & 782.7 & & 19455.7 \\
\hline $1^{4} \Sigma_{3 / 2}^{+}$ & (5)3/2 & 18763.6 & 5.525 & 22.4 & 693.1 & & 19456.7 \\
\hline $1^{4} \Pi_{5 / 2}$ & (1) $5 / 2$ & 17132.6 & 4.640 & 24.0 & 2324.6 & & 19457.2 \\
\hline
\end{tabular}

a NIST [18].

b Meyer and Bohn [4], spin-free states.

c Brue and Hutson [3].

d Shao et al. [5].

The calculations of the vibrational states energies and the Franck-Condon factors are performed using the LEVEL program package [28]. The perturbations of the vibrational states, which belong to different electronic terms with the equal $\Omega$ values (for example, $1^{2} \Pi_{1 / 2}$ and $2^{2} \Sigma_{1 / 2}^{+}$) and overlap in some region of energies, were not calculated.

\section{Results and discussion}

\subsection{Electronic states}

The calculated PECs are performed in Fig. 1 and are also given in the Supplementary Material. The calculated molecular spectroscopic constants (the electronic term energy $T_{e}$, the equilibrium internuclear distance $R_{e}$, the state binding energy $D_{e}$ and the harmonic vibrational frequency $\omega_{e}$ ) are presented in Table 1 . Table 1 also shows the results of the $a b$ initio calculations [3-5].

As there is no experimental values of spectroscopic parameters for ground and excited states to determine the accuracy of the calculations in Table 1 we compare the calculated energies of molecular states at the dissociation limits (at the internuclear distance of $17.0 \AA$ ) with the sum of the experimental energies [18] of separated atoms. The energies obtained for the $1^{2} \Pi_{3 / 2}, 1^{2} \Pi_{1 / 2}$, and $2^{2} \Sigma_{1 / 2}^{+}$states corresponding to the limit II are $11178.0,11728.7$, $11732.3 \mathrm{~cm}^{-1}$ and with the SOC splitting of $554.3 \mathrm{~cm}^{-1}$. These data are in agreement with the experimental ones (see Table 1 ).

Our calculated equilibrium internuclear distance $\left(R_{e}=5.763 \AA\right)$ for the $X^{2} \Sigma_{1 / 2}^{+}$state higher than Brue and Hutson's [3] (5.144 $\AA$ ), Shao's [5] (5.161 $\AA$ ) and Meyer and Bohn's [4] (5.657 $\AA$ ) values, but is similar to the last one. Our calculated dissociation energy of the ground $X^{2} \Sigma_{1 / 2}^{+}$state $\left(D_{e}=158.7 \mathrm{~cm}^{-1}\right)$ is close to the Meyer and Bohn's value [4] $\left(182 \mathrm{~cm}^{-1}\right)$ and significantly lower than Shao's [5] $\left(542 \mathrm{~cm}^{-1}\right)$ and Brue and Hutson's ones [3] $\left(621 \mathrm{~cm}^{-1}\right)$ (see Section 1). The shallow potential well is caused by weak polarizability of the $6 \mathrm{~s}$ state of Cs atom and signals the van der Waals' character of the molecule. In contrast to the ground state PEC, the first excited states $\left(1^{2} \Pi_{1 / 2}, 1^{2} \Pi_{3 / 2}\right.$, and $\left.2^{2} \Sigma_{1 / 2}^{+}\right)$have deeper wells due to stronger polarizability of the $6 \mathrm{p}$ state of cesium atom.

During the calculations the $1^{2} \Delta_{1 / 2,3 / 2}$ states that should belong to limit III disappeared, the remaining ones being shifted and SOC splitting of this limit being overestimated by $1633.8 \mathrm{~cm}^{-1}$. This may be caused by the not including f-electrons of $\mathrm{Yb}$ atom in active space through the calculations.

The term, related to limit IV, must appear according to experimental data between fine-structure components of limit V. However, within the calculations such a situation did not arise. "Intruded" term $5^{2} \Sigma_{1 / 2}^{+}$caused a perturbation and as a result dropped down by $2739.8 \mathrm{~cm}^{-1}$ and affected terms belonging to limit III.

The PECs obtained for limit V are shifted down approximately by $253 \mathrm{~cm}^{-1}$ in comparison with NIST energies [18]. Nevertheless, the total SOC splitting of this limit equals to $2422.5 \mathrm{~cm}^{-1}$, which almost coincides with experimental one $\left(2421.9 \mathrm{~cm}^{-1}\right)$.

Fig. 2 presents the calculated TDMs as the functions of internuclear distances for the transitions from the ground state to the all spin-mixed excited states. We concerned only the transitions allowed by $\Delta \Omega=0, \pm 1$ selection rules. In the area of the dissociation limits only TDMs related to the limit II are nonzero due to fulfilling the selection rules for the atomic transitions.

Since in photoassociation processes diatomic molecules (atomic pairs) are formed in weakly-bounded vibrational states with large internuclear distances near the dissociation limit, the value of the TDM can be significant for the probabilities of the optical exciting at the first step of the two-photon optical scheme for the producing ultracold $\mathrm{CsYb}$ molecules in the ground rovibronic state. At the large internuclear distances for the transitions from the ground electronic state to the all excited electronic states under consideration (with the exception of $2^{2} \Sigma_{1 / 2}^{+}, 1^{2} \Pi_{1 / 2}$, and $1^{2} \Pi_{3 / 2}$ states, i.e. states of the limit II) TDMs are zero (see Fig. 2). Therefore, it is assumed that namely these exited states $\left(2^{2} \Sigma_{1 / 2}^{+}, 1^{2} \Pi_{1 / 2}\right.$, and $1^{2} \Pi_{3 / 2}$ ) are of more direct interest for the production of ultracold $\mathrm{CsYb}$ molecules by optical methods. 


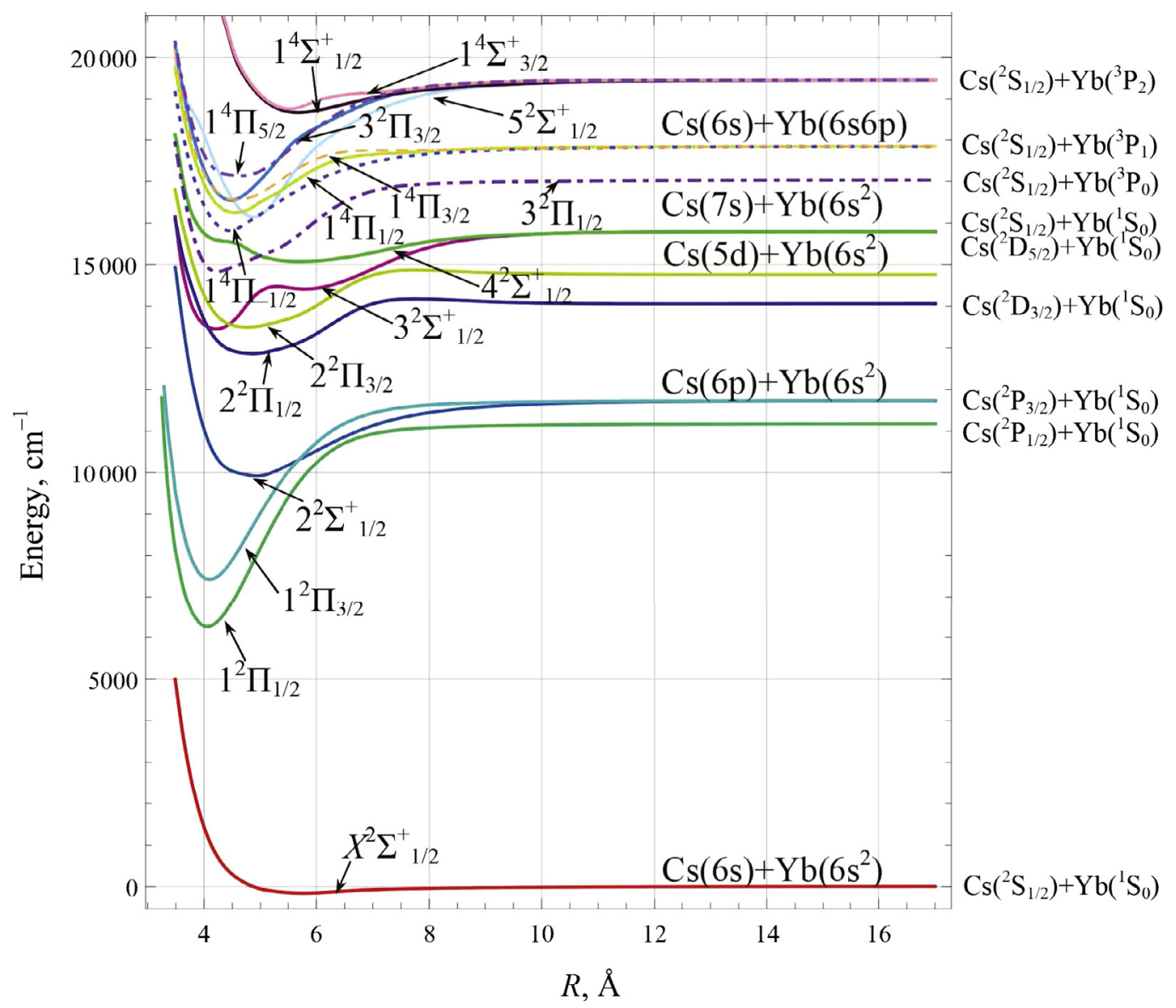

Fig. 1. The low-lying terms of the CsYb molecule calculated at the CASSCF(3,12)/XMCQDPT2 + SOC level of theory.

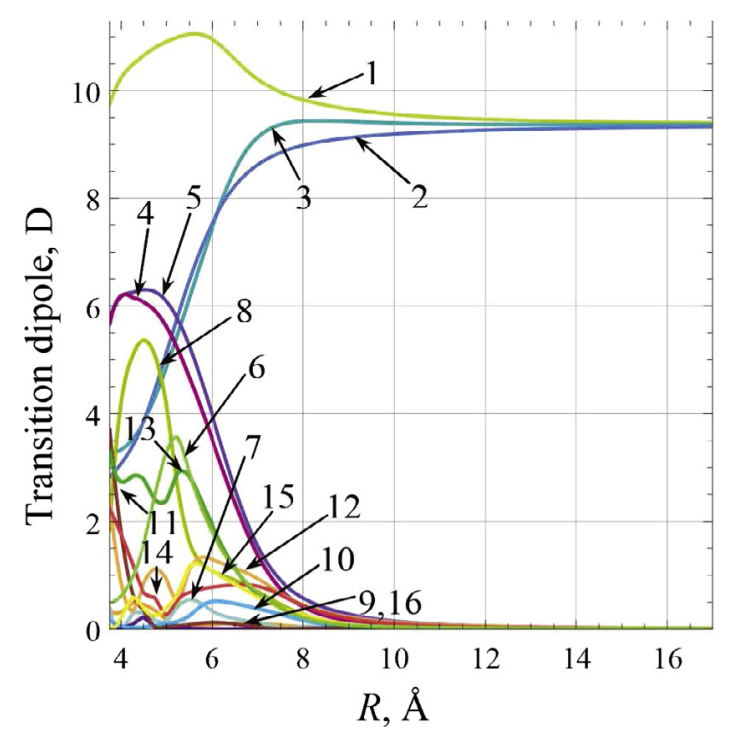

Fig. 2. Calculated transition dipole moments for the transitions from the ground (1) $1 / 2$ state to the states (2)1/2 (1), (1)3/2 (2), (3) $1 / 2$ (3), (4) $1 / 2(4),(2) 3 / 2(5),(5) 1 / 2$ (6), (6)1/2 (7), (7)1/2 (8), (1)-1/2 (9), (8)1/2 (10), (3)3/2 (11), (9) $1 / 2$ (12), (4)3/2 (13), (10) $1 / 2(14),(5) 3 / 2(15)$, and (1) $5 / 2(16)$.

\subsection{Vibrational states}

For calculations of vibrational energies and frequencies reduced mass of the molecule is important. The $\mathrm{Yb}$ atom has seven stable isotopes: ${ }^{168} \mathrm{Yb} \quad(0.13 \%),{ }^{170} \mathrm{Yb}(3.04 \%),{ }^{171} \mathrm{Yb}(14.28 \%),{ }^{172} \mathrm{Yb}$ (21.83\%), ${ }^{173} \mathrm{Yb}(16.13 \%),{ }^{174} \mathrm{Yb}(31.83 \%)$ and ${ }^{176} \mathrm{Yb}(12.76 \%)$. For Cs atom there is only one $\left({ }^{133} \mathrm{Cs}\right)$ stable isotope. We calculate the vibrational energies for ${ }^{133} \mathrm{Cs}^{174} \mathrm{Yb}$ molecule, as the most abundant isotopomer, and some other isotopomers for all spin-mixed PECs up to the dissociation limit of the corresponding state, and then we obtained the harmonic vibrational frequencies $\omega_{e}$ for these electronic states (see Table 1 ).

For the ground $X^{2} \Sigma_{1 / 2}^{+}$state and excited $1^{2} \Pi_{1 / 2}, 1^{2} \Pi_{3 / 2}$, and $2^{2} \Sigma_{1 / 2}^{+}$ states we obtained 37, 145, 137 and 120 vibrational levels, respectively. Fig. 3, as an example, shows vibrational energies $E_{\mathrm{v}}$ and vibrational intervals $\Delta G\left(v^{\prime}+1 / 2\right)$ for the ground $X^{2} \Sigma_{1 / 2}^{+}$state and "shelflike" $3^{2} \Sigma_{1 / 2}^{+}$state. Kinks on such dependencies (see Fig. 3c, d) usually indicate the existence of some peculiarities on the potential curves: several minima, flat areas, etc. Since the highly excited vibrational states located near the dissociation limit of the ground $X^{2} \Sigma_{1 / 2}^{+}$state are of primary concern to optical production of the ultracold molecules, we used approximation function $-C_{3} / r^{3}-C_{6} / r^{6}$ to reproduce more proper values of bound states of the ground electronic state near the threshold starting from $11 \AA$. The coefficients $C_{3}$ and $C_{6}$ are $6496.53 \mathrm{~cm}^{-1} \AA^{3}$ and $1.60325 \times 10^{7} \mathrm{~cm}^{-1} \AA^{6}$, respectively. With this outer part of the ground state PEC we already obtained 45 bound vibrational states, and well depth became $160.8 \mathrm{~cm}^{-1}$. The insert in Fig. 3a shows differences in sequences of vibrational energies calculated without and with asymptotic function. For other excited states $\left(2^{2} \Pi_{1 / 2}, 2^{2} \Pi_{3 / 2}, 3^{2} \Sigma_{1 / 2}^{+}, 4^{2} \Sigma_{1 / 2}^{+}, 3^{2} \Pi_{1 / 2}, 1^{4} \Pi_{-1 / 2}, 1^{4} \Pi_{1 / 2}, 2^{2} \Pi_{3 / 2}, 5^{2} \Sigma_{1 / 2}^{+}\right.$, $1^{4} \Pi_{3 / 2}, 1^{4} \Sigma_{1 / 2}^{+}, 1^{4} \Sigma_{3 / 2}^{+}$, and $\left.1^{4} \Pi_{5 / 2}\right)$ we obtained $55,57,134,85$, $110,111,89,131,147,44,59,78$, and 115 bound vibrational states, respectively. 


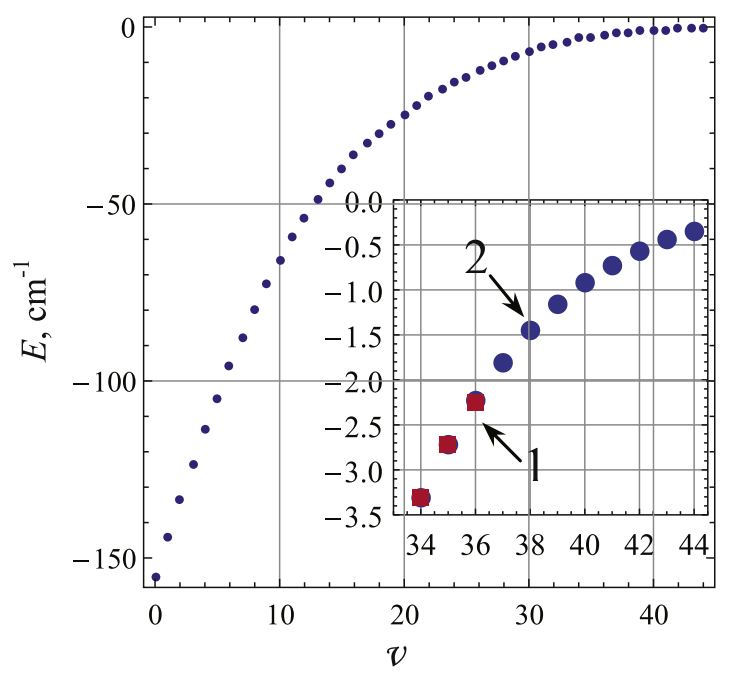

$a$

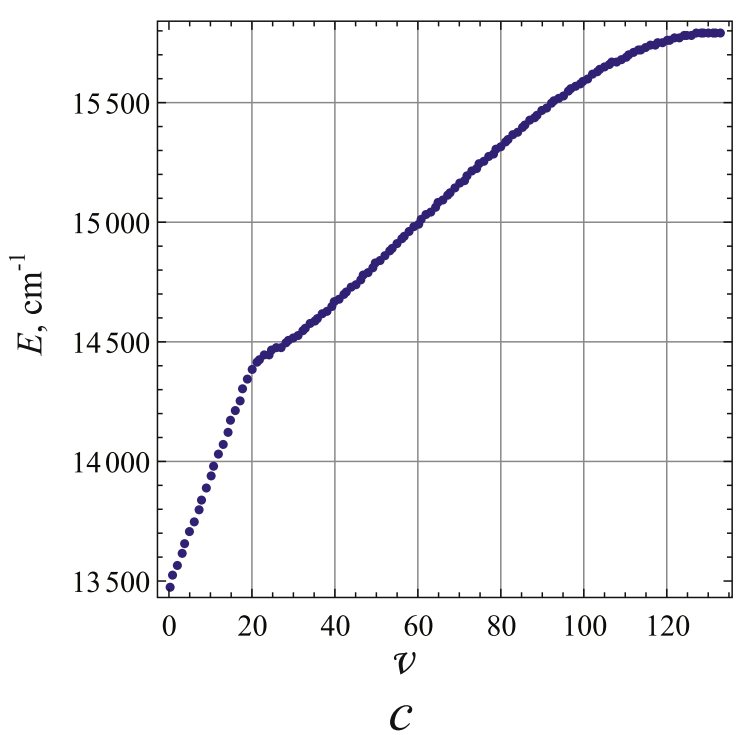

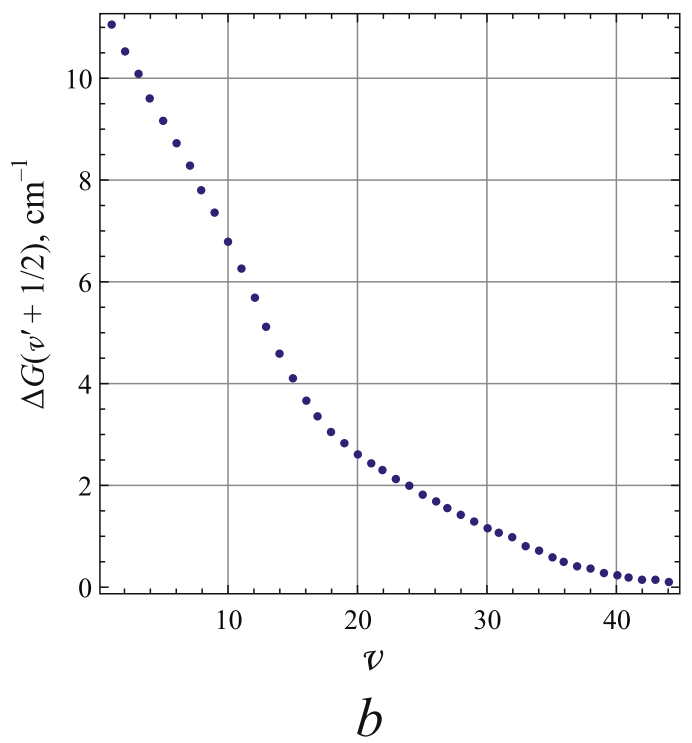

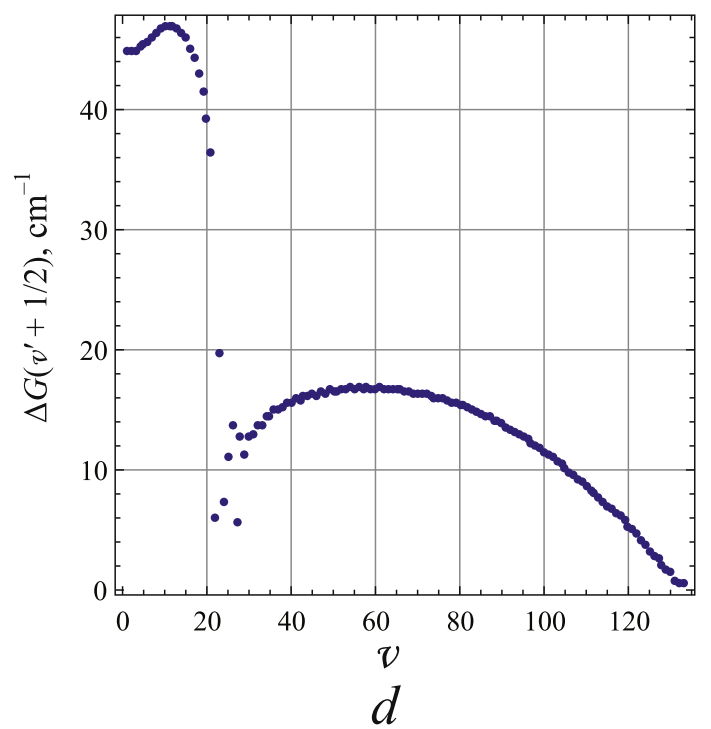

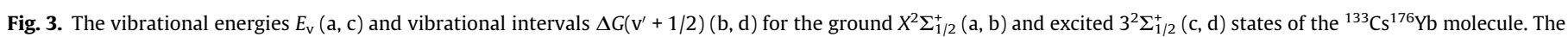
insert in a shows sequences of vibrational energies near dissociation limit calculated without (1) and with asymptotic function (2).

\subsection{Franck-Condon factors}

On the basis of calculated PECs we obtain the Franck-Condon factors (FCFs) between the pointful states of the ${ }^{133} \mathrm{Cs}^{174} \mathrm{Yb}$ molecule. Using the $\Delta \Omega=0, \pm 1$ selection rules we have calculated FCFs for vibronic transitions between the spin-mixed electronic states. We concern the transitions between the fixed vibrational level $\mathrm{v}^{\prime}$ of the upper electronic state and a sequence of vibrational levels $\mathrm{V}^{\prime \prime}$ of the lower electronic state applying the selection rules $\Delta J=0$ or $\Delta J= \pm 1$.

The FCFs obtained for transitions between the vibrational levels of the first three exited electronic states and the vibrational levels of the ground electronic state with $\Delta J=0$ selection rule are presented in Fig. 4. FCFs shown here are calculated for the transitions from the lowest vibrational levels $\left(v^{\prime}=0 \ldots 4\right)$ of the excited electronic states (Fig. 4a, d, g) (1), for the transitions with the largest FCFs to the ground rovibronic $X^{2} \Sigma_{1 / 2}^{+}\left(v^{\prime \prime}=0, J=0\right)$ state (Fig. $4 \mathrm{~b}$, e, h) (2), and for the transitions with the largest values of all possible FCFs (Fig. 4c, f, i) (3).
The FCFs distributions for the $X^{2} \Sigma_{1 / 2}^{+} \leftarrow 1^{2} \Pi_{1 / 2}$ and $X^{2} \Sigma_{1 / 2}^{+} \leftarrow 1^{2} \Pi_{3 / 2}$ transitions have the same character (see Fig. $4 \mathrm{a}-\mathrm{f}$ ). The largest values of FCFs for the transitions to the "absolute" ground state $X^{2} \Sigma_{1 / 2}^{+}\left(\mathrm{v}^{\prime \prime}=0, J=0\right)$ are about 0.07 with $\mathrm{v}^{\prime}=70 \ldots 74$ for $1^{2} \Pi_{1 / 2}$ state and $v^{\prime}=61 \ldots 65$ for $1^{2} \Pi_{3 / 2}$ state. The rovibronic transitions between weakly-bound vibrational states near the dissociation limits of the excited electronic states $\left(v^{\prime}=139 \ldots 144\right.$ for $1^{2} \Pi_{1 / 2}$ state and $v^{\prime}=130 \ldots 137$ for $1^{2} \Pi_{3 / 2}$ state) and the ground electronic state $\left(\mathrm{v}^{\prime \prime}=40 \ldots 46\right)$ have the largest probabilities.

For the $X^{2} \Sigma_{1 / 2}^{+} \leftarrow 2^{2} \Sigma_{1 / 2}^{+}$transitions to the "absolute" ground state $X^{2} \Sigma_{1 / 2}^{+}\left(v^{\prime \prime}=0, J=0\right)$ the largest FCFs occur for the transitions from the $2^{2} \Sigma_{1 / 2}^{+}\left(v^{\prime}=13 \ldots 21\right)$ vibronic states with FCFs values of about 0.1 . According to predicted average TDM value of about 9.5 $\mathrm{D}$ in the 3.5-17.0 $\AA$ region for the $2^{2} \Sigma^{+} \leftarrow X^{2} \Sigma^{+}((3) 1 / 2 \leftarrow(1) 1 / 2)$ transition (see Fig. 2), the $2^{2} \Sigma_{1 / 2}^{+}$(or (3)1/2) state is concerned to be a suitable candidate for two-photon optical scheme for the producing $\mathrm{CsYb}$ molecules in the ground rovibronic state. 

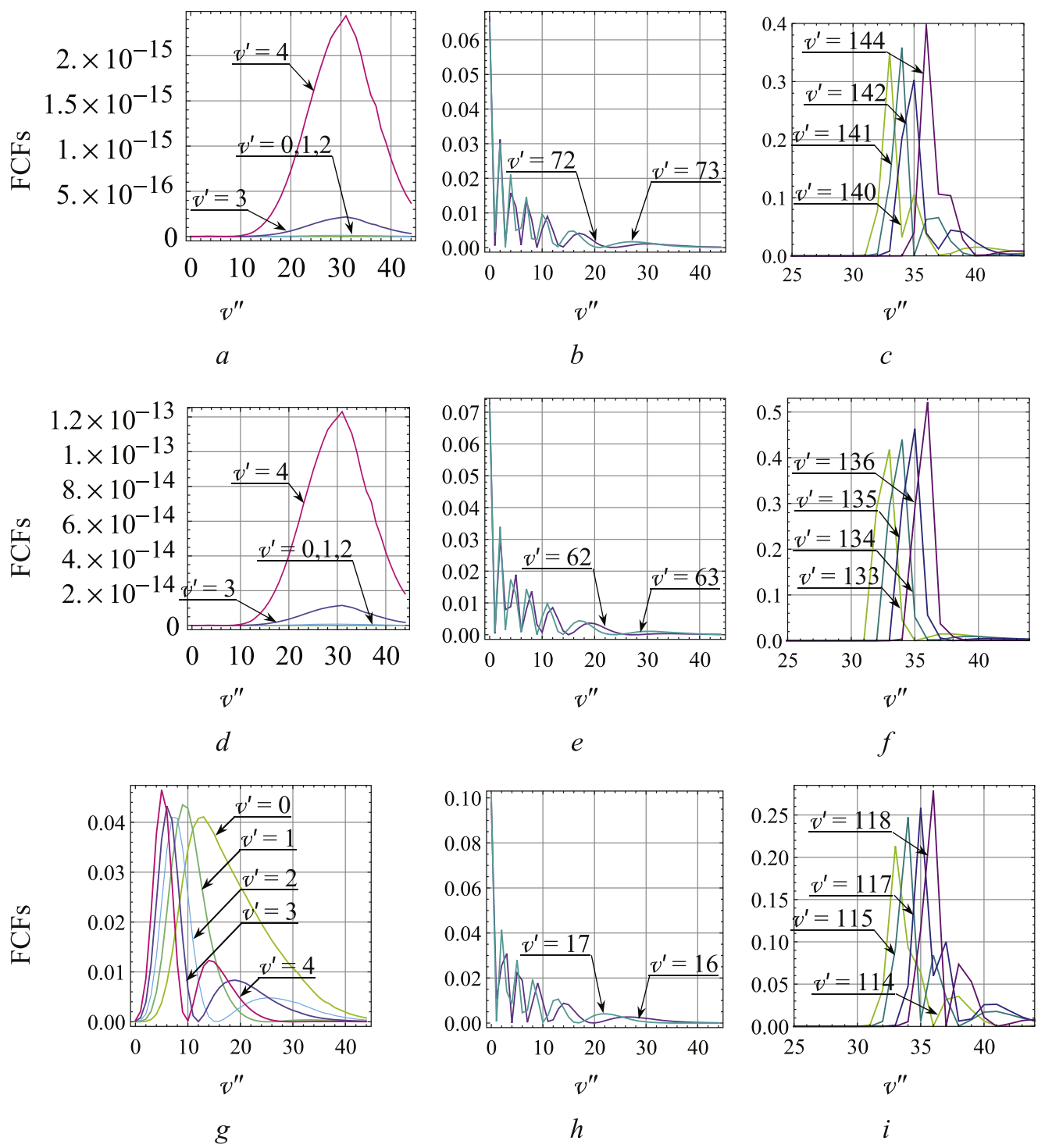

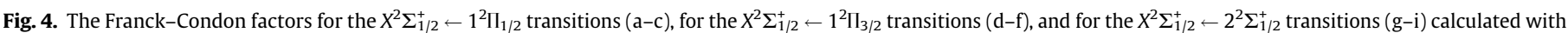
the $\Delta J=0$ selection rule for the ${ }^{133} \mathrm{Cs}^{176} \mathrm{Yb}$ molecule.

\section{Conclusions}

The system of the low-lying electronic states of the CsYb molecule taking into account the spin-orbit coupling at the high level of theory was calculated for the first time. PECs of the ground and 16 excited states were constructed, for each of them the system of vibrational levels and vibrational frequencies were computed. The value of $158.7 \mathrm{~cm}^{-1}$ (or $160.8 \mathrm{~cm}^{-1}$ with approximation function) obtained for dissociation energy of the ground state indicates van der Waals' character of the molecule. The transition dipole moments were calculated, Franck-Condon factors for vibronic transitions were determined. Since at the $\mathrm{Cs}(6 \mathrm{p})+\mathrm{Yb}\left(6 \mathrm{~s}^{2}\right)$ dissociation limit our obtained PECs are in a good agreement with the experimental [18] energies and since the calculations for the $\mathrm{KRb}$ [17] and RbYb [12] molecules in the same approximation show accurate values, we expect the results of our current calculations for the ground and low-lying excited states of the CsYb molecule to be close to the correct values. The effective schemes (optical cycles, for example) for transferring CsYb molecules to the ground rovibronic state by initial excitation into overlying states can be developed on the basis of the calculated spectral and dynamic characteristics. The results obtained can be used for spectroscopic studies of CsYb molecule, as well as for the synthesis of such molecules. The ytterbium-containing diatomics (such as RbYb and SrYb) now are considered as perspective molecular systems for the experiments under cold and ultracold conditions [13,29]. We assume that the molecule under consideration also can be used for the purposes mentioned.

\section{Acknowledgments}

The authors are grateful to Dr. Alexander Granovsky (Firefly Project, Moscow, Russia) for him helpful discussions.

\section{Appendix A. Supplementary material}

Supplementary data associated with this article can be found, in the online version, at http://dx.doi.org/10.1016/j.comptc.2017.03. 046. 


\section{References}

[1] O. Dulieu, C. Gabbanini, The formation and interactions of cold and ultracold molecules: new challenges for interdisciplinary physics, Rep. Prog. Phys. 72 (2009) 086401.

[2] G. Quéméner, P.S. Julienne, Ultracold molecules under control!, Chem Rev. 112 (2012) 4949-5011.

[3] D.A. Brue, J.M. Hutson, Prospects of forming ultracold molecules in ${ }^{2} \Sigma$ states by magnetoassociation of alkali-metal atoms with Yb, Phys. Rev. A 87 (2013) 052709.

[4] E.R. Meyer, J.L. Bohn, Electron electric-dipole-moment searches based on alkali-metal- or alkaline-earth-metal-bearing molecules, Phys. Rev. A 80 (2009) 042508.

[5] Q. Shao, L. Deng, X. Xing, D. Gou, X. Kuang, H. Li, Ground state properties of the polar alkali-metal-ytterbium and alkaline-earth-metal-ytterbium molecules: a comparative study, J. Phys. Chem. A 121 (2017) 2187-2193.

[6] S.N. Tohme, M. Korek, R. Awad, Ab initio calculations of the electronic structure of the low-lying states for the ultracold LiYb molecule, J. Chem. Phys. 142 (2015) 114312.

[7] G. Gopakumar, M. Abe, B.P. Das, M. Hada, K. Hirao, Relativistic calculations of ground and excited states of LiYb molecule for ultracold photoassociation spectroscopy studies, J. Chem. Phys. 133 (2010) 124317.

[8] P. Zhang, H.R. Sadeghpour, A. Dalgarno, Structure and spectroscopy of ground and excited states of LiYb, J. Chem. Phys. 133 (2010) 044306.

[9] S. Kotochigova, A. Petrov, M. Linnik, J. Kłos, P.S. Julienne, Ab initio properties of Li-group-II molecules for ultracold matter studies, J. Chem. Phys. 135 (2011) 164108.

[10] S.N. Tohme, M. Korek, Electronic structure with vibration-rotation study of the NaYb molecule, Chem. Phys. Lett. 638 (2015) 216-226.

[11] S.N. Tohme, M. Korek, Electronic structure calculation of the KYb molecule with dipole moments, polarizabilities, and ro-vibrational studies, Comput. Theor. Chem. 1078 (2016) 65-71.

[12] M.B. Shundalau, A.A. Minko, Ab initio multi-reference perturbation theory calculations of the ground and some excited states of the YbRb molecule, Comput. Theor. Chem. 1103 (2017) 11-16.

[13] L.K. Sørensen, S. Knecht, T. Fleig, C.M. Marian, Four-component relativistic coupled cluster and configuration interaction calculations on the ground and excited states of the RbYb molecule, J. Phys. Chem. A 113 (2009) 12607-12614.

[14] S.N. Tohme, M. Korek, Electronic structure and rovibrational calculation of the low-lying states of the RbYb molecule, Chem. Phys. 410 (2013) 37-44.

[15] M. Borkowski, P.S. Zuchowski, R. Ciuryło, P.S. Julienne, D. Kẹdziera, Ł. Mentel, P. Tecmer, F. Münchow, C. Bruni, A. Görlitz, Scattering lengths in isotopologues of the RbYb system, Phys. Rev. A 88 (2013) 052708.
[16] A.A. Granovsky, Extended multi-configuration quasi-degenerate perturbation theory: the new approach to multi-state multi-reference perturbation theory, J. Chem. Phys. 134 (2011) 214113.

[17] M.B. Shundalau, G.A. Pitsevich, A.E. Malevich, A.V. Hlinisty, A.A. Minko, R. Ferber, M. Tamanis, Ab initio multi-reference perturbation theory calculations of the ground and low-lying electronic states of the $\mathrm{KRb}$ molecule, Comput. Theor. Chem. 1089 (2016) 35-42.

[18] NIST Atomic Spectra Database, http://www.nist.gov/pml/data/asd.cfm (accessed 07.02.17)

[19] Y. Wang, M. Dolg, Pseudopotential study of the ground and excited states of $\mathrm{Yb}_{2}$, Theor. Chem. Acc. 100 (1998) 124-133.

[20] I.S. Lim, P. Schwerdtfeger, B. Metz, H. Stoll, All-electron and relativistic pseudopotential studies for the group 1 element polarizabilities from $\mathrm{K}$ to element 119, J. Chem. Phys. 122 (2005) 104103.

[21] S.-W. Chang, H.A. Witek, Choice of optimal shift parameter for the intrude state removal techniques in multireference perturbation theory, J. Chem. Theory Comput. 8 (2012) 4053-4061.

[22] M.B. Shundalau, A.A. Minko, Determination of the optimal energy denominator shift parameter of $\mathrm{KRb}$ electronic states in quantum chemical computations using perturbation theory, J. Appl. Spectrosc. 82 (2016) 901904.

[23] W.R. Wadt, An approximate method to incorporate spin-orbit effects into calculations using effective core potentials, Chem. Phys. Lett. 89 (1982) 245 248

[24] S. Koseki, M.W. Schmidt, M.S. Gordon, MCSCF/6-31G(d, p) calculations of oneelectron spin-orbit coupling constants in diatomic molecules, J. Phys. Chem. 96 (1992) 10768-10772.

[25] S. Koseki, M.S. Gordon, M.W. Schmidt, N. Matsunaga, Main group effective nuclear charges for spin-orbit calculations, J. Phys. Chem. 99 (1995) 12764 12772.

[26] Alex A. Granovsky, Firefly version 8. http://classic.chem.msu.su/gran/firefly/ index.html (accessed 07.02.17).

[27] M.W. Schmidt, K.K. Baldridge, J.A. Boatz, S.T. Elbert, M.S. Gordon, J.H. Jensen, S Koseki, N. Matsunaga, K.A. Nguyen, S. Su, T.L. Windus, M. Dupuis, J.A. Montgomery Jr., General atomic and molecular electronic structure system, J. Comput. Chem. 14 (1993) 1347-1363.

[28] R.J. Le Roy, Level 8.0: A Computer Program for Solving the Radial Schroedinger Equation for Bound and Quasibound Levels, University of Waterloo Chemical Physics Research Report CP-663, 2007.

[29] M. Tomza, F. Pawłowski, M. Jeziorska, C.P. Koch, R. Moszynski, Formation of ultracold SrYb molecules in an optical lattice by photoassociation spectroscopy: theoretical prospects, Phys. Chem. Chem. Phys. 13 (2011) 18893-18904. 